\title{
Abiding Geocast for Warning Message Dissemination in Vehicular Ad Hoc Networks
}

\author{
Qiangyuan $\mathrm{Yu}^{1,2}$ \\ ${ }^{1}$ College of Computer Science and Technology \\ Jilin University \\ No. 2699, QianJin Street, Changchun, 130012, P.R.China \\ qiangyuan@jlu.edu.cn
}

\author{
Geert Heijenk $^{2}$ \\ ${ }^{2}$ Faculty of EEMCS / DACS \\ University of Twente \\ P.O. Box 217, 7500 AE Enschede, The Netherlands \\ geert.heijenk@utwente.nl
}

\begin{abstract}
Vehicular ad hoc networks (VANETs) are emerging as a new network environment for intelligent transportation systems (ITS). In many applications envisaged for VANETs, traffic information needs to be disseminated to a group of relevant vehicles and maintained for a duration of time. Here a system of abiding geocast is presented for disseminating warning message among mobile vehicles in VANETs. In this system vehicles on a stretch of roads carry safety messages to vehicles in the opposite direction, so as to warn them regarding a dangerous situation ahead. The objective is that as much as possible all relevant vehicles receive the warning before they reach the warning line, whereas as few messages are broadcasted as possible. Opposite vehicles are preferred as relays to reduce broadcast overhead and help message delivery upstream. Furthermore, wait time of individual relay vehicles are set dynamically for the next broadcast, thus unnecessary broadcasts can be saved while keeping the warning message in the affected area. Simulations show the quality of the proposed system by measuring the broadcast overhead under various conditions.
\end{abstract}

Keywords-abiding geocast; warning message dissemination; vehicular ad hoc networks

\section{INTRODUCTION}

Vehicular ad hoc networks (VANETs) is becoming a promising field of research and can play an important role in intelligent transportation systems (ITS), which can enable many applications, such as traffic monitoring and message dissemination. VANETs are based on short-range wireless communication (e.g., IEEE 802.11) between vehicles, and are constructed on-the-fly and do not require any investment besides the wireless network interfaces that will be a standard feature in the next generation of vehicles. These vehicles will be capable to run network protocols that will exchange messages for safer and more fluid traffic on the roads.

Avoiding accidents and traffic jams are two main immediate benefits of vehicular networks. For instance, drivers could benefit from receiving real-time alerts about accidents happening at a short distance in front of their vehicles. When an accident occurs, warning messages must be transmitted to inform all relevant vehicles. Because the warning message will be valid for a period of time, it should be continuously disseminated around the event for a specific time interval.

The problem addressed in this paper is: How to ensure that a traffic warning message is disseminated with high probability and low overhead to all the potentially affected vehicles? It is the main goal of abiding geocast to disseminate information about accidents or congestions to every vehicle that will pass through the warning zone during the lifetime of the event. It uses multihop ad-hoc networking to ensure that any node passing the region will receive the message, up until the message lifetime expires, or it is explicitly revoked. The idea in our system is that vehicles on a stretch of road carry warning message to vehicles in the opposite direction, so as to warn them regarding a dangerous situation ahead. The objective is that as much as possible all vehicles in the opposite direction receive the warning before they reach the event, whereas as few messages are broadcasted as possible. The problem can be described by Fig. 1. There is an emergency event occurring on a road, the initial vehicle $s$ know it first, then vehicle $s$ will try to inform the vehicles approaching the emergency event.

We emphasis two points: abiding geocast and disseminate in time. As illustrated by Fig. 1, we try to inform all vehicles moving left before they pass the safety line during the lifetime of event, at the same time vehicles broadcast as few messages as possible. The notion of in-time means vehicles need to be notified before pass the safety line, not as soon as possible.

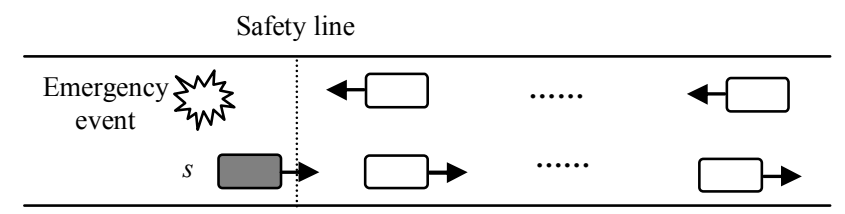

Figure 1. Warning message dissemination

\section{RELATED WORK}

Following the high interest and potential of inter-vehicle communications (IVC), some research groups have explored the idea of information dissemination using IVC. Some approaches appeared addressing different types of applications and environments, which are designed according to different criteria. For the case of safety messages dissemination, broadcast is the preferred mechanism. Several strategies have been suggested to improve the simple flooding approach.

RBM (Role-Based Multicast) aims to achieve maximum reachability in a sparsely connected network by using the storecarry-forward mechanism [1]. The nodes start broadcasting the alert only when ensured of the existence of neighbors

This research is sponsored by the National Natural Science Foundation of China under Grant No. 60703022. 
reachable. However, RBM requires that each node maintain a list of all its neighbors, and the maintenance will generate additional overhead. In [2] two schemes, TRADE and DDT, are used to improve the broadcast in the context of the road transport. The protocol IVG (Inter-Vehicles Geocast) achieves to inform all the vehicles in risk area of a highway about any danger [3]. These works have considered passing information only through vehicles traveling in the same direction, rather than taking advantage of traffic in opposite direction lanes.

ODAM (Optimized Dissemination of Alarm Messages) restricts re-broadcast to only special relays and in risk zones [4]. It allows overcoming problems as fragmentation, reliability and neighbors' determination, and adopts periodic rebroadcasts of the alarm messages by introducing dynamic relays. Another approach for safety applications makes use of simple directed broadcast-based communication without considering the efficiency of the message dissemination mode [5]. An emergency message dissemination protocol for IVC divides the highway into virtual cells [6], which move as the vehicles move. Another cluster-based protocol disseminates traffic and road conditions by using a combination of MANET and DTN (Delay Tolerant Networking) methodologies [7]. In [8] a contention based strategy is used to disseminate a message among vehicles in road traffic. The multi-hopping is performed by all vehicles up to a threshold number of hops and does not depend on the lane direction of the vehicle. A formal model of data dissemination in VANETs is proposed to study how VANET characteristics affect the performance of data dissemination [9]. The results show how opposite vehicles can be exploited as carriers to quickly disseminate information to the vehicles that follow. None of above works, however, allow for information to be maintained in an area for a specified time.

Abiding geocast is introduced to disseminate information to a group of nodes in an area for a duration of time [10]. It employs periodic flooding or epidemic dissemination. When a vehicle enters such an area, the virtual warning sign is displayed for the driver. An efficient protocol is proposed to minimize the number of broadcasts needed for maintaining a regional alert over a period of time [11], but the protocol is only discussed under discrete time and location.

\section{PROPOSED SYSTEM}

The abiding geocast for message dissemination is described in this section. First some assumptions of the system are presented. Then the dissemination strategies in our system are discussed. Finally message form and algorithm are given.

\section{A. System Assumptions}

Vehicles should be equipped with a device enabling it to obtain its location at any time. In this system, vehicles are equipped with omnidirectional radio antennas of transmission range $R$. Communications between vehicles are supposed to be bidirectional, and are based on the broadcasting of messages.

When an emergency situation occurs, one vehicle which detects this problem starts broadcasting a warning message to inform the other relevant vehicles about the danger. Here we consider the system with following assumptions:
- Given a bidirectional stretch of road.

- Vehicles move throughout the road with constant speed randomly chosen within an allowable range.

- Only one initiator of disseminating, other vehicles act as relays.

- Only one active warning message to be disseminated.

The warning information is characterized by the event topic, content, location, safety distance, and time limit, etc, where safety distance means that vehicles moving towards the event should be informed at least distance away from the event and time limit is the validity of the warning event.

\section{B. Dissemination strategies}

In our system, we mainly use two strategies to improve the system efficiency. In order to overcome fragmentation, we mainly utilize vehicles traveling in opposite direction as relays to reduce broadcasts and help message delivery upstream. In order to save unnecessary broadcasts while keeping the warning message in the affected area, we dynamically set the wait time of individual relay vehicles for the next broadcast when it receives (directly or indirectly) a message from other vehicles travelling in the same direction.

Here, we use the notion of effect line to indicate beyond which point vehicles will become inactive and not broadcast any more, whereas effect distance is the distance between safety line and effect line. The area between these two lines is called effect area of an active vehicle. The vehicles will enter the road from two ends as a Poisson distribution with traffic volume $\lambda$ (vehicle/hour). As a consequence of the Poisson distribution, the headways between consecutive vehicles are distributed exponentially with mean 3600/ $\lambda$. Each vehicle runs independently and maintains a constant speed chosen randomly in the interval $\left[S_{\text {mean }}-\varepsilon, S_{\text {mean }}+\varepsilon\right]$, here $S_{\text {mean }}$ is the speed mean and $\varepsilon$ is the speed variation. In order to guarantee that the warning message is not lost, effect distance has to be set large enough so that there is at least one relay left before one vehicle leaves the effect area. Effect distance is only set by the beginner of dissemination, and then it will be constant and delivered to other vehicles with the message. Effect distance should be valued larger than the maximum possible interval between consecutive vehicles, and we use effect distance factor $\gamma$ to set its value as (1). According to the simulation result, we will choose the minimum $\gamma$ that can make the message kept in the affected area during its lifetime.

$$
\text { effect distance }=S_{\text {mean }} * 3600 / \lambda * \gamma
$$

A vehicle will become active and act as a relay after it knows the warning, and a relay is responsible for broadcasting the message to other relevant vehicles. When one vehicle becomes active, it need not broadcast immediately and continuously. How to set the wait time depends on some conditions: a) moving direction and speed of the sender and receiver; b) relative position of the sender and receiver; c) distance to the safety line and effect line. 


\section{1) Overcoming fragmentation}

When vehicles are out of transmission range, commonly known as fragmentation, it is impossible to deliver the message immediately, especially in sparse networks. Normally one vehicle has to broadcast frequently because another vehicle in the same direction may move into and out of transmission range quickly, the overhead is high because many periodic broadcasts do not reach any new vehicles. In order to overcome fragmentation and disseminate efficiently, in our system, a relay vehicle is only responsible for delivering the message to vehicles travelling in the opposite direction. To guarantee opposite vehicles are informed, the relay only has to broadcast frequent enough so that no other vehicles move into, pass, and leave its transmission range between successive broadcasts. This time interval of successive broadcasts is much larger than for two vehicles traveling in the same direction that move into each other's transmission range momentarily.

The wait time of one active vehicle for the next broadcast is set according to transmission range $R$, its speed $S_{\text {self }}$, current location Curr_loc, location of safety line and maximum speed of vehicles with the conservative assumption that the vehicle is moving at the maximum allowable speed $S_{\max }$. The wait time can be set for vehicles leaving and approaching the event using (2) and (3) respectively, during the time, opposite vehicles can not travel from beyond the transmission range, pass then leave the range of broadcasting vehicle or cross the safety line.

$$
\begin{array}{r}
\text { WaitTime }=\min \left((R+\mid \text { Curr_Loc }- \text { Safetyline } \mid) / S_{\max },\right. \\
\left.2 * R /\left(S_{\max }+S_{\text {self }}\right)\right)
\end{array}
$$

$$
\text { WaitTime }=2 * R /\left(S_{\max }+S_{\text {self }}\right)
$$

\section{2) Updating the wait time dynamically}

Here we use the strategy that one active vehicle can wait for some time without periodically broadcasting if it knows another vehicle moving in the same direction is active now and broadcasting periodically. At any time, one relay vehicle is enough in one moving direction. In Fig. 2 (a), when vehicle $r$ directly receives the message from vehicle $s$ travelling in its front, vehicle $r$ can wait until vehicle $s$ move out of the effect area, even vehicle $r$ maybe overtake $s$ and pass the effect during its wait time.

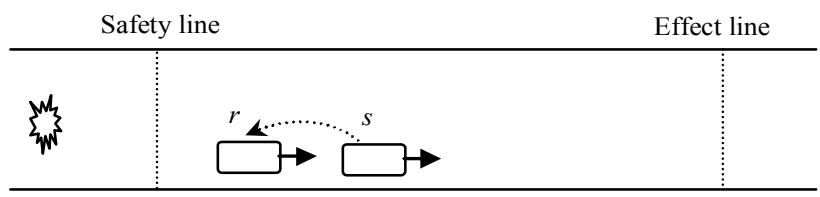

a) Receives message from front vehicle directly

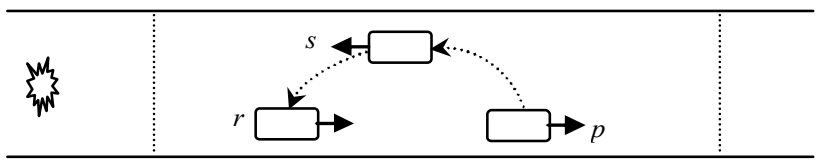

b) Receives message from front vehicle indirectly

Figure 2. Update wait time when receives message from front vehicles
In a sparse network, a vehicle rarely receives message from other vehicles with the same direction. In order to indirectly supply receivers the information of other vehicles with the same direction through opposite vehicles, in our system, each vehicle records the information of its last opposite vehicle when it receives a message, and also sends this information when broadcasting. When one vehicle receives a message from an opposite vehicle, this one will become its new last-opposite vehicle. When it receives a message from another vehicle with the same direction, it will inherit the last-opposite vehicle from the sender. So, the information about the last-opposite vehicle will be updated with the received messages. This information includes the location, speed, direction, and send-time of the last-opposite vehicle. In Fig. 2 (b), at first vehicle $p$ delivers a message to vehicle $s$, then vehicle $r$ receives a message from opposite vehicle $s$, and $r$ knows $p$ is in its front and active now, so vehicle $r$ can wait until vehicle $p$ moves out of the effect area. In this case, receiver will calculate the wait time with location of effect line or safety line, actual location and speed of sender's last-opposite vehicle.

When updating the wait time, one vehicle should guarantee that the new value of wait time is bigger than the old one, namely the value of timer will monotonically increase.

\section{Message form and algorithm}

In the algorithm of our system we use a timer to control the next broadcast of active vehicles. When the timer triggers, the vehicle will broadcast after first checking its current location and time considering the time limit of event, location of safety line or effect line. After broadcasting, the vehicle will set its timer for the next broadcast. The message form is listed in Fig. 3 and the algorithm for receiver is given in Fig. 4.

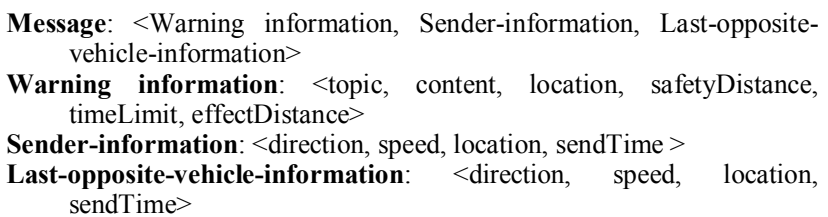

Figure 3. Message form

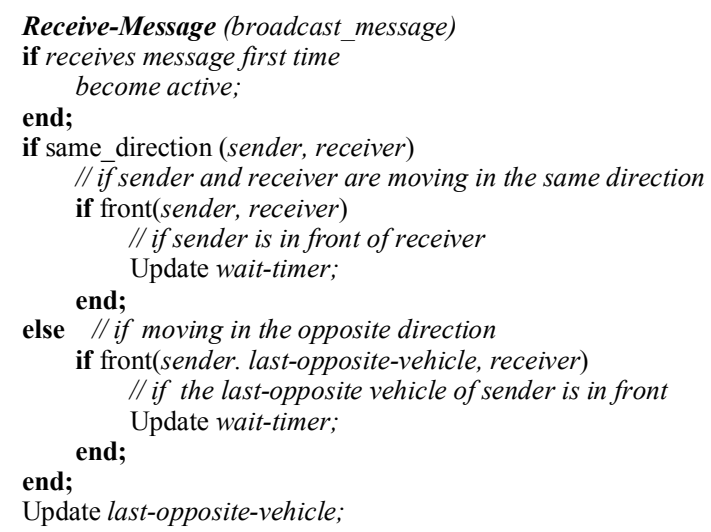

Update last-opposite-vehicle;

Figure 4. Algorithm for vehicle receiving message 


\section{EXPERIMENTS}

In order to evaluate the performance of our system we created a mobility model to simulate the vehicle behavior on the road. This mobility model is implemented by means of the OMNET++ simulator $[12,13]$, a discrete event simulation environment and the mobility framework plug-in [14], which supports node mobility, dynamic connection management and a wireless channel model. In our model, we use IEEE 802.11 in the Mac layer and the transmission range is limited to $250 \mathrm{~m}$.

We evaluate the model under various conditions using broadcast overhead as a metric. Existing methods for abiding geocast are few, and have different assumptions, so comparison is not possible. When considering the transmission range, only the one-dimensional distance along the road is considered and the road's width is neglected.

\section{A. Simulation Setup}

The parameters of our model are listed in Table I. For all the simulations in this paper, we fix the length of the straight road to $6 \mathrm{~km}$, and assume one vehicle can overtake other vehicles freely. The location of the warning event is at 0 meters, and the safety distance is 500 meters. For all the runs, simulation time is $4000 \mathrm{~s}$, the start time of the warning event is at $400 \mathrm{~s}$, and it will last to $4000 \mathrm{~s}$ with the valid duration 60 minutes. We omit the first 400 s to let the system reach a stable state considering the distribution of vehicles over the road. When the warning event occurs, the beginner of dissemination is at the location of the safety line.

TABLE I. PARAMETERS

\begin{tabular}{|l|l|}
\hline \multicolumn{1}{|c|}{ Description } & \multicolumn{1}{c|}{ Value } \\
\hline transmission range $(\mathrm{R})$ & $250 \mathrm{~m}$ \\
\hline safety distance & $500 \mathrm{~m}$ \\
\hline $\begin{array}{l}\text { speed mean }\left(S_{\text {mean }}\right) \\
\text { speed variation } \varepsilon\end{array}$ & $30 \mathrm{~m} / \mathrm{s}$ \\
\hline traffic volume $\lambda$ & $200 \sim 1000(\mathrm{veh} / \mathrm{hr})$ \\
\hline effect distance factor $\gamma$ & $6.0 \sim 9.0$ \\
\hline
\end{tabular}

We run the model with various parameter settings, and for each parameter setting 30 simulation runs are done, to achieve statistically reliable results. The error bars in the result figures represent a $95 \%$ confidence interval.

\section{B. Simulation runs and results}

We evaluate the performance of the warning message dissemination model under different scenarios; mainly check the role of two parameters $\gamma$ and $\lambda$ on the broadcast overhead.

\section{1) Simulation $I$}

Firstly we will check how the effect distance factor $\gamma$ influences the broadcast overhead. From the result in Fig. 5, we can see that a smaller $\gamma$ will cause higher probability of warning message loss as showed in Table II. With the traffic volume varying from 200 to $1000 \mathrm{veh} / \mathrm{hr}$, our model can guarantee no warning message loss when $\gamma$ is over 8.0. As we see from Fig. 5 , the average broadcast per informed vehicle declines with $\lambda$ and increases with $\gamma$. This means that the broadcast overhead in a dense network is lower than that in a sparse network, because one vehicle in a dense network will receive message from other vehicles with high probability, then it can wait and save broadcasts. Increasing $\gamma$ will result in more broadcasts, because vehicles will broadcast more before passing the effect line and stop broadcasting. When $\gamma$ is equal to or more than 8 , we can keep the warning message in the affected area during its lifetime. Note that we do not study delivery time as a performance metric, since we are interested in delivery before a vehicle cross the safety line. The delivery ratio is $100 \%$ if the effect distance factor $\gamma$ is sufficiently large (see Table II).

Fig. 6 shows that the total broadcast overhead, i.e., for all vehicles, is about 0.6 times per second for varying traffic volume. The overhead less depends on traffic volume, so this model is practicable in dense and sparse network. Compared with the capacity of wireless LAN, the overhead is low. We also differ the wait times of multi receivers with their distances to the sender, so the chance of collision is very small.

TABLE II. IMPACT OF EFFECT DISTANCE FACTOR ON MESSAGE LOSS

\begin{tabular}{|c|c|c|c|c|}
\hline $\begin{array}{c}\text { effect distance } \\
\text { factor } \boldsymbol{\gamma}\end{array}$ & 6.0 & 7.0 & 8.0 & 9.0 \\
\hline $\begin{array}{c}\text { probability of } \\
\text { message loss }\end{array}$ & $8.15 \%$ & $0.74 \%$ & 0 & 0 \\
\hline
\end{tabular}

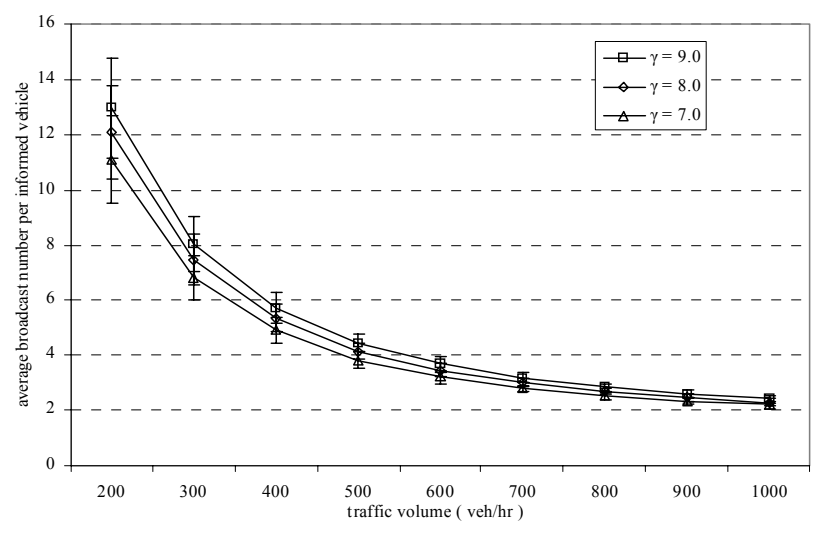

Figure 5. Broadcast overhead under different effect distance factor $\gamma$

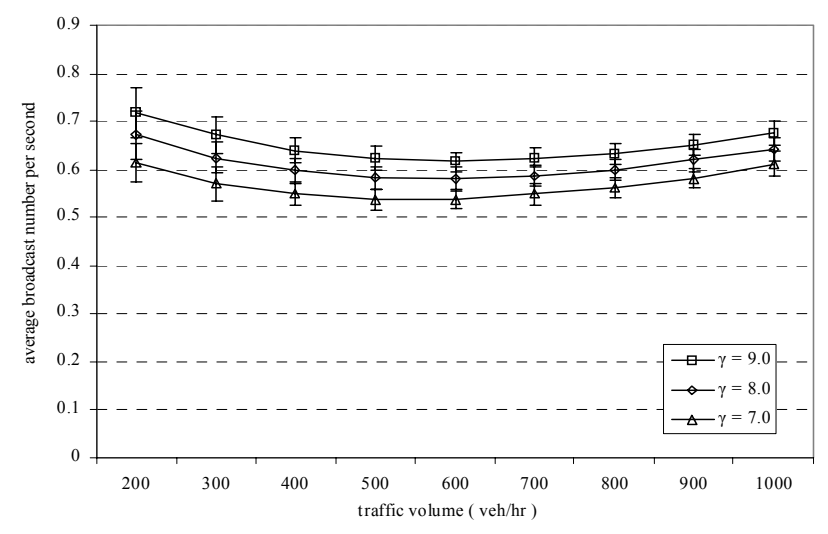

Figure 6. Broadcast overhead in unit time 


\section{2) Simulation II}

Now we aim to see the variation of broadcast overhead along with dissemination time. We fix the effect distance factor $\gamma$ to 8.0 , so there is no warning message loss in this setting.

As we see from Fig. 7, the broadcast overhead can reach the stable state in a short time, and the initial stage takes about 10 minutes after dissemination begins.

At the same time, we can check the location where the vehicles approaching the event are informed for the first time. We can see from Fig. 8 that most affected vehicles are informed when they just enter the effect area by passing the effect line, i.e. far away from the safety line. Thus the drivers of these affected vehicles will have enough time to make a decision before reaching the warning event.

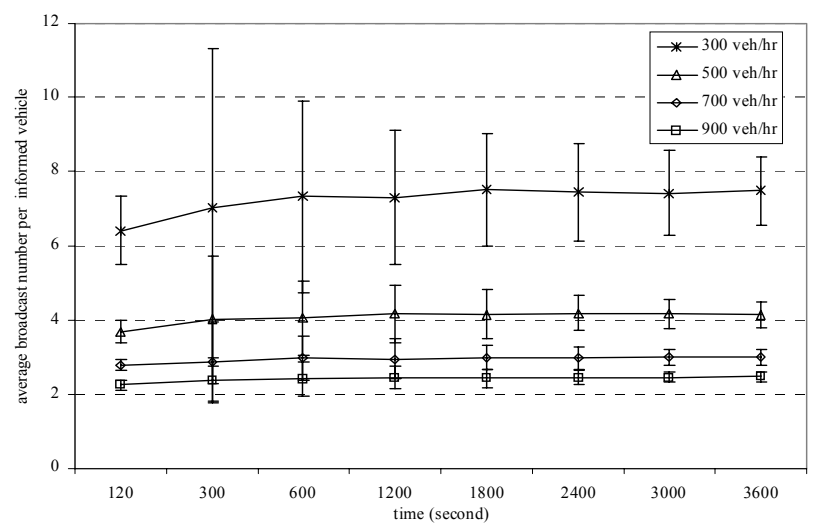

Figure 7. Variation of broadcast overhead with dissemination time

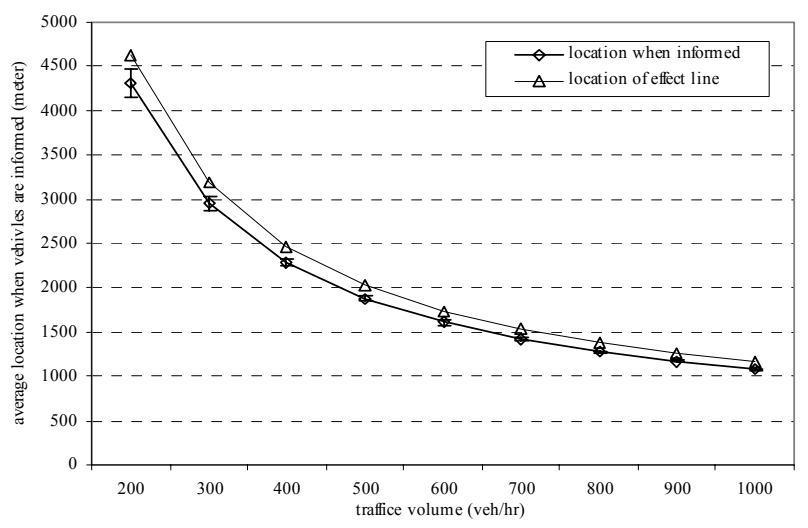

Figure 8. Location where vehicles are informed for the first time

\section{CONCLUSION AND FUTURE WORK}

We have introduced a novel time-stable geocast system for disseminating warning messages in VANETs. We utilize two strategies to improve the efficiency of disseminating: vehicles traveling in opposite direction are used as preferred relays to overcome fragmentation and reduce broadcasting. Dynamic wait time setting is adopted to save unnecessary broadcasting while keeping the warning message in the effect area. The record of last-opposite vehicle is the key for one vehicle to get information of other vehicles moving in the same direction, especially in sparse networks. In order to investigate the performance of this approach, a mobility model has been developed. We observed the performance of the model with respect to various parameter settings. Results show that this model can successfully inform affected vehicles in a sparse network with few broadcasts in unit time as well as in a dense one. Also the disseminating process can reach the stable state quickly after dissemination begins, and affected vehicles are informed earlier when they just cross the effect line.

Ongoing and future research includes the prediction of vehicle mobility. We plan to predict the average headway between consecutive vehicles, although it is given to the beginner vehicle in our model now. Meanwhile, by analytical calculation and by microscopic simulation of freeway traffic with a given percentage of vehicles equipped for inter-vehicle communication, we investigate how the equipment level influences the efficiency of information propagation.

\section{REFERENCES}

[1] L. Briesemeister, G. Hommel, "Role-based multicast in highly mobile but sparsely connected ad hoc networks", in Proceedings of the First Annual Workshop on Mobile Ad Hoc Networking and Computing, August 2000, pp. 45-50.

[2] M. Sun, W. Feng, T. Lai, K. Yamada, H. Okada, K. Fujimura, "GPSBased Message Broadcasting for Intervehicle Communication", in Proceedings of the 2000 International Conference on Parallel Processing, 2000, pp. 2685-2692

[3] Bachir and A. Benslimane, "A Multicast protocol in ad hoc networks inter-vehicle geocast", in Proceedings of the 57th IEEE Semiannual Vehicular Technology Conference, vol. 4, April 2003, pp. 2456-2460.

[4] Benslimane, "Optimized Dissemination of Alarm messages in Vehicular Ad-Hoc Networks (VANET)", in Proceedings of the 7th IEEE International Conference HSNMC 2004, France, pp. 655-666.

[5] Q. Xu, T. Mak, J. Ko and R. Sengupta, "Vehicle-to vehicle safety messaging in DSRC", in Proceedings of the First ACM workshop on Vehicular ad hoc Networks (VANET), 2004.

[6] M. Durresi, A. Durresi and L. Barolli, "Emergency Broadcast Protocol for Inter-Vehicle Communications", in Proceedings of the 11th International Conference on Parallel and Distributed Systems (ICPADS '05), 2005, pp. 402-406.

[7] T. D. C. Little, A. Agarwal, "An information propagation scheme for VANETs", in Proceedings of IEEE Intelligent Transportation Systems, 2005, pp. 155-160.

[8] L. Briesemeister, L. Schafers and G. Hommel, "Disseminating Messages among Highly Mobile Hosts based on Inter-Vehicle Communication", in Proceedings of IEEE Intelligent Vehicles Symposium,2000, pp.522-527.

[9] T. Nadeem, P. Shankar and L. Iftode, "A Comparative Study of Data Dissemination Models for VANETs", in Proceedings of the 3rd Annual International Conference on Mobile and Ubiquitous Systems, July 2006.

[10] Christian Maihofer, Tim Leinmuller, and Elmar Schoch, "Abiding geocast: time-stable geocast for ad hoc networks", in Proceedings of the 2nd ACM international workshop on Vehicular ad hoc networks (VANET '05), New York, USA, 2005, pp. 20-29.

[11] Q. Sun, and H. Garcia-Molina, "Using Ad-hoc Inter-vehicle Networks for Regional Alerts", Technical Report, Stanford University, 2004.

[12] OMNET++ Community Website. http://www.omnetpp.org/.

[13] Andras Varga, "The OMNET++ Discrete Event Simulation System", in Proceedings of the European Simulation Multiconference, 2001.

[14] W. Drytkiewicz, S. Sroka, V. Handziski, A. Koepke, and H. Karl. "A Mobility Framework for OMNeT++", in Proceedings of the 3rd International OMNeT++ Workshop, Hungary, January 2003. 